\title{
Effects of Different Inoculated Pathogens on Microbial Species and Abundances of Rhizosphere Soil of Tobacco
}

\author{
Zebin Chen", a Xi Gao ${ }^{2}$, Li Lin", Yuan Su${ }^{3}$, Jiani Liu', Lei Yu4, Dingkang Wang ${ }^{1}$, Shengguang \\ $\mathrm{Xu}^{5, \mathbf{b}, *}$ \\ ${ }^{1}$ College of Agronomy, Kunming University, Kunming, Yunnan Province, China. \\ ${ }^{2}$ College of Plant Protection, Yunnan Agricultural University, Kunming, Yunnan Province, China. \\ ${ }^{3}$ Key Laboratory of Characteristic Biological Resource Development and Utilization of Colleges and \\ Universities in Yunnan Province, Kunming, Yunnan Province, China. \\ ${ }^{4}$ Yunnan Urban Characteristic Agriculture Engineering Technology Research Center, Kunming, Yunnan \\ Province, China. \\ ${ }^{5}$ Yunnan Institute of Biological Carbon Engineering Research Center, Kunming, Yunnan Province, China. \\ azbchenkmu@163.com, bsgxu2011@126.com \\ *Corresponding author
}

Keywords: high throughput sequencing; tobacco; rhizosphere soil; root-knot nematode; black shank

\begin{abstract}
The aim of this research was to clarify the effects of different inoculated pathogens on microbial species and abundances of rhizosphere soil of tobacco. The rhizosphere soil of root-knot nematode and Phytophthora parasitica infected tobacco were collected and detected. The DNA of 16S rDNA-V4 region and ITS1 region in the rhizosphere soil of infected tobacco were sequenced through high throughput sequencing technique; the abundance and diversity of microorganisms were analyzed. In the 6S rDNA-V4 region and ITS1 region, 152815 and 61505 original sequences were detected respectively. After filtering low quality sequences, 147558 and 60109 effective sequences were obtained, which could be divided into 6871 and 796 OTUs. The diversity analysis showed that pathogen inoculation caused significant differentiation in the microbial community of rhizosphere soil. The Shannon index and Chao1 index significantly increased compared with the control group, indicating that pathogen inoculation could significantly increase microbial diversity and abundance. The research on microbial diversity of rhizosphere soil of tobacco after pathogen inoculation can provide reference for the development of remediation technology on soil ecology.
\end{abstract}

\section{Introduction}

Tobacco belongs to Solanaceae family of Dicotyledoneae class; it is one of the most important economic crops in China, and occupies an important position in the agricultural economy. [1] But in recent years, black shank disease of tobacco and its combination with root-knot nematode infection sharply reduce the yield and quality of tobacco, and even cause extinction; tobacco farmers suffered huge economic losses. [2] The comprehensive control of tobacco black shank disease and root-knot nematode infection is essential for the sustainable development of tobacco. [3] Nematodes lead to physiological changes of host plants, making them more vulnerable to fungal infection. [4] Root-knot nematodes infected tobacco plants are susceptible to other root pathogens, especially fungi. [5] Studies have shown that microbiological control methods could be used to improve the rhizosphere microenvironment of plants, and reduce disease to a certain extent. [6] In this study, high throughput sequencing technology was used to study the spices of microorganisms in rhizosphere soil of tobacco after pathogen inoculation. The numbers and compositions of rhizosphere microorganisms of healthy and infected tobacco plants were also analyzed. This paper could provide scientific basis for the control of tobacco black shank disease and root-knot nematode infection through micro-ecological techniques. 


\section{Research Material and Research Methods}

\subsection{Tobacco varieties and pathogen inoculation.}

The tobacco variety was Yunyan 202. The root damage perfusion method was used to inoculate Phytophthora parasitica, which can cause black shank disease of tobacco. First, a small amount of sterile water was used to moisten the culture soil; then sterilized forceps were inserted deeply around the tobacco plant to achieve the effect of root injury. The $1 \times 10^{4} \mathrm{cfu} / \mathrm{mL}$ zoospore suspension liquid was used to inoculate tobacco plants at 6-8 stages; each plant was inoculated with $10 \mathrm{~mL}$ suspension liquid. Clear water was used in another group for control. [7] Root knot nematode was obtained from the root knot nematode infected tobacco plants in the root knot nematode nursery of Yuxi Research and Experimental Base in Yunnan. The soil was washed away from the root of infected tobacco plants; the egg sacs were removed with tweezers, and then put into the $1 \%$ sodium hypochlorite for 3 minutes to sterilize. Then egg sacs were washed with sterile water for 3 times, and placed in petri dishes with a small amount of aseptic water at 25 degrees centigrade. Hatched root knot nematodes were collected every 24 hours, and the nematode suspension was put into the sterilized triangle bottles with aseptic water. The concentration was about 2000 nematode/mL. After the seedlings were transplanted for 3 days, each plant was irrigated with $200 \mathrm{~mL}$ suspension. Clear water was used in another group for control. [8]

\subsection{Collection and preservation of soil samples.}

In the squaring stage of tobacco, the "S" sampling method was used to collect soil samples from the rhizosphere soil of tobacco plants at the depth of $5-20 \mathrm{~cm}$. The mixed soil samples were packed in sterile plastic bags, sealed and brought back to the laboratory for refrigeration at $4^{\circ} \mathrm{C}$. [9] Soil sample of the control group was numbered as AB3-2; soil sample of root-knot nematode inoculated plants was numbered as AG3-2; soil sample of Phytophthora parasitica inoculated plants was numbered as AH3-2.

\subsection{Extraction of total DNA and PCR amplification.}

DNA extraction kit for soil produced by FastDNA was used to extract soil DNA. PCR was amplified by specific primers of 16S rDNA-V4 and ITS1 regions. [10]

\subsection{High throughput sequencing of PCR products.}

High throughput sequencing of PCR products was entrusted to TinyGene Bio-Tech (Shanghai) Co. Ltd.

\subsection{Alpha diversity analysis.}

Mothur software was used in Alpha diversity analysis; Coverage index was employed to reflect the coverage of sample library. Chao1 algorithm was used to estimate the diversity index of OTU number in the community; Shannon index was used to measure the heterogeneity of community. Corresponding formula were as follows. The formula of Coverage index: $C=1-n_{1} / \mathrm{N}$. In this formula, $\mathrm{n}_{1}$ was the number of OUT which contained only one sequence; $\mathrm{N}$ was the total number of sequences. The Shannon index was calculated as $\mathrm{H}^{\prime}=-\Sigma \mathrm{P}_{\mathrm{i}} \times \ln \mathrm{P}_{\mathrm{i}}$. In the formula, Pi was the ratio of each varity to the total species. The formula of Chao1 index: $S_{\text {chao1 }}=S_{\text {obs }}+n_{1}\left(n_{1}-1\right) / 2\left(n_{2}+1\right)$. In this formula, $S_{\text {chao1 }}$ was the number of estimated OTU; $\mathrm{S}_{\text {obs }}$ was the actual OTU number; $\mathrm{n}_{1}$ was the number of OTU which had only one sequence; $\mathrm{n}_{2}$ was the number of OTU which had two sequences. [11]

\section{Results and Analysis}

\subsection{Sequencing results and sequencing depth verification.}

By sequencing the 16S rDNA-V4 region, 152815 original sequences were detected from the 3 soil samples. After filtering low quality sequences, the total number was 147558. After removing redundant sequences, 142976 effective sequences were obtained. Under the similarity level of 97\%, 
the sequences were divided into 6871 OTUs. The Shannon index for bacteria was highest in the sample of root-knot nematode infected tobacco $\left(\mathrm{AG}_{3-2}\right)$, indicating that inoculation of root-knot nematode can improve the bacterial diversity of tobacco rhizosphere soil. The OTU number and Chao1 index of root-knot nematode inoculated tobacco $\left(\mathrm{AG}_{3-2}\right)$ were significantly higher than those of the control group, indicating that the inoculation of root-knot nematode can increase the diversity and abundance of bacteria in the rhizosphere soil. The Shannon index for bacteria in the rhizosphere soil of tobacco which inoculated Phytophthora parasitica $\left(\mathrm{AH}_{3-2}\right)$ was also slightly increased, indicating that the infection of Phytophthora parasitica can also improve the diversity of bacterial community in the rhizosphere soil of tobacco. The Chao1 index was slightly higher than the control group, indicating that the infection of Phytophthora parasitica increased the total abundance of bacteria in the rhizosphere soil.

By sequencing the ITS1 region, 92838 original sequences were detected from the 3 soil samples. After filtering low quality sequences, the number became 63733. After removing redundant sequences, 63661 effective sequences were obtained. Under the similarity level of 97\%, the sequences were divided into 820 OTUs. The Shannon index for fungi in the rhizosphere soil of root-knot nematode infected tobacco $\left(\mathrm{AG}_{3-2}\right)$ and Phytophthora parasitica infected tobacco $\left(\mathrm{AH}_{3-2}\right)$ were significantly lower than that of the control group, indicating that the infection of different pathogens could not improve the diversity of fungal communities in the rhizosphere soil of tobacco. The OTU number and Chao1 index for fungi in the rhizosphere soil of root-knot nematode infected tobacco $\left(\mathrm{AG}_{3-2}\right)$ were significantly lower than those of the control group, indicating that infection of root-knot nematodes decreased the diversity and abundance of fungi in the rhizosphere soil of tobacco.

\subsection{The distribution characteristics of bacterial and fungal communities.}

Dominant bacteria in the rhizosphere soil of tobacco under three treatment methods were distributed into 4 phyla, namely Proteobacteria, accounting for $69.02 \%$, Crenarchaeota, accounting for $13.68 \%$, Actinobacteria, accounting for $8.37 \%$ and Bacteriodetes, accounting for $4.24 \%$. Compared with the control group, in the root-knot nematode inoculated tobacco, the proportions Rhodoplanes and Kaistobacter decreased; almost no Thermomonas, Marinobacter, Alcanlvorax and Rhodoplanes were undetected. The proportions of Candidates Nitrososphaera and Pseudoncardia increased; the proportions of Streptomyces and Hydrogenophaga remained at low level. Compared with the control group, in Phytophthora parasitica infected tobacco, the proportions of Marinobacter, Candidates Nitrososphaera, Rhodoplanes, Alcanlvorax, Pseudoncardia, and Streptomyces reduced; the proportions of Hydrogenophaga, Kaistobacter and Thermomonas increased significantly.

Compared with the control group, in root-knot nematode inoculated tobacco, the proportions of Corynascella, Chaetomium, Sordariales and Chaetomiaceae increased, while the proportions of Aspergillus, Tremellomycetes, Mortierella and Penicillium reduced. Compared with the control group, in Phytophthora parasitica infected tobacco, the proportions of Aspergillus, Chaetomium and Tremellomycetes decreased, while the proportions of Chaetomiaceae and Mortierella increased. In addition, the proportions of Corynascella, Retroconis, Sordariales and Penicillium remained unchanged.

\section{Discussion and Conclusion}

By sequencing and analyzing 16S rDNA-V4 region and ITS1 region, it was found that compared with the control group, the species and abundance of microbes in root-knot nematode infected group and Phytophthora parasitica infected group significantly changed. The inoculation of root-knot nematode lead to greatest changes. The analysis of $16 \mathrm{~S}$ rDNA-V4 region sequencing results showed that the coverage rates of the control group, root-knot nematode infected group and Phytophthora parasitica infected group were $95.2 \%, 95 \%$ and $96.2 \%$ respectively, indicating that the sequencing results could reflect the diversity and abundance of bacteria in the real environment. The Shannon index of the root-knot nematode infected group was higher than that of the control group, while the Shannon index of Phytophthora parasitica infected group was lower than that of the control group, 
indicating that bacterial diversity changes in rhizosphere soil after inoculation were related to the species of inoculated pathogens. Comparing root-knot nematode infection with Phytophthora parasitica inoculation, it was found that the proportions of Marinobacter, Alcanlvorax, Kaistobacter and Thermomonas in the former were lower than those in the latter; while the proportions of Rhodoplanes, Streptomyces, Hydrogenophaga, Candidates Nitrososphaera and Pseudoncardia in the former were higher than those in the latter. It could be found that the effects of root-knot nematode inoculation on the abundance of bacteria were greater than those of Phytophthora parasitica inoculation.

Through sequencing fungi in the ITS1 region, it was found that the coverage rates of the control group, root-knot nematode infected group and Phytophthora parasitica infected group were 98.7\%, $98.6 \%$ and $98.6 \%$ respectively, indicating that the sequencing results could reflect the diversity and abundance of fungi in the real environment. The Shannon index of the root-knot nematode group was lower than that of the control group, while the Shannon index of the Phytophthora parasitica infected group was higher than that of the control group, indicating that the fungal diversity changes in rhizosphere soil after inoculation were related to the species of inoculated pathogens. Analyzing OTUs in the rhizosphere soil of each group from the perspectives of phylum, class, order, family and genus, and further exploring the composition of communities in samples, following facts were found. The abundance of Retroconis did not change after the inoculation of two pathogens; the abundance of Pulvinula changed. The effects of root-knot nematode inoculation on the species and abundance of fungi were far greater than the effects of Phytophthora parasitica inoculation, which indirectly indicated that root-knot nematodes could cause more serious damage in the micro ecology of rhizosphere soil of tobacco.

Through annotated analysis of 16S rDNA-V4 region and ITS1 region, it was found that the decrease or loss of Thermomonas, Marinobacter, Alcanlvorax, Rhodoplanes and Pulvinula was related to root-knot nematode infection. The decreasing abundance of Marinobacter, Candidates Nitrososphaera and Rhodoplanes, the increasing abundance of Hydrogenophaga, Kaistobacter, Thermomonas and Mortierella, especially the absence of Pulvinula, were related to black shank disease of tobacco.

\section{Acknowledgements}

This paper is supported by National Natural Science Foundation of China (41361056, 31460491, 31660426); Joint Special Fundamental Research of Local Universities in Yunnan Province (2017FH001-041, 2017FH001-035, 2017FH001-005); Talent Introduction Program of Kunming University (YJL14005); Key Disciplines (Ecology) Project of Yunnan Education Department (05000511311); Open Fund Project of Key Laboratory of Characteristic Biological Resources Development and Utilization in Yunnan Province (GXKZ201716); China National Tobacco Corp Science and Technology Project (2018530000241020, 2018530000241016).

\section{References}

[1] A.W. Frederick, Tobacco Diseases and Decays, Duke University Press, North Carolina, 1957.

[2] C.Y. Lai, Agricultural Plant Pathology, Science Press, Beijing, 2003.

[3] H. Zhao, D.L. Peng, J.L. Zhu, et al., Reviews on the root-knot nematodes, J. Plant Protection. 29 (2003).

[4] M.L. Zhu, Advances in the biological control of root-knot nematodes in tobacco, J. Microbiology China. 31 (2004).

[5] L.Q. Dong, K.Q. Zhang, Microbial control of plant-parasitic Nematodes: a five-party interaction, J. Plant Soil. 288 (2006).

[6]W. Tan, Y.H. Wu, Tobacco Pathology, China Agriculture Press, Beijing, 2003. 
[7] R.C. Edgar, B.J. Haas, J.C. Clemente, et al., UCHIME improves sensitivity and speed of chimera detection. J. Bioinformatics. 27 (2011).

[8] R.C. Edgar, UPARSE: highly accurate OTU sequences from microbial amplicon reads, J. Nature Methods. 10 (2013).

[9] Q. Wang, G.M. Garrity, J.M. Tiedje, et al., Naive Bayesian classifier for rapid assignment of rRNA sequences into the new bacterial taxonomy, J. Applied and Environmental Microbiology. 73 (2007).

[10] T.Z. DeSantis, P. Hugenholtz, N. Larsen, et al., Greengenes, a chimera-checked 16S rRNA gene database and workbench compatible with ARB, J. Applied and Environmental Microbiology. 72 (2006).

[11] Z.B. Chen, B. Li, D.K. Wang, et al., Study on the diversity of endophytic bacteria in maize using Illumina MiSeq high throughput sequencing system, J. Modern Food Science and Technology. 32 (2016). 